\title{
Remote Estimation of Leaf Water Content Using Spectral Index Derived From Hyperspectral Data
}

\author{
Chengfang Zhang, Zhiyuan Pan \\ Department of Architectural Engineering \\ Wuhan University of Technology Huaxia College \\ Wuhan, 430070, China \\ E-mail:ruoruozcf@163.com
}

\author{
Heng Dong*, Fangjian He,Xingbang $\mathrm{Hu}$ \\ College of Resources and Environment Engineering \\ Wuhan University of Technology \\ Wuhan, 430070, China \\ E-mail:dongheng1986@163.com
}

\begin{abstract}
Monitoring crop water content by remote sensing has been an important problem for agricultural drought monitoring and water resources management. The triangular water vegetation index is affected by canopy structure parameters, such as leaf area index. To improve this, we used the method of ratio index to put forward a new vegetation index with the improved soil adjustable vegetation index. The precision of the new index for monitoring agricultural drought was verified by simulated data and field observation data. The results showed that the new index was a good method to monitoring the crop water content. However, due to the limitations of data acquisition, the field validation was only with the data of wheat which observed in Yucheng, shandong province. In the future, more tests still need to be done.
\end{abstract}

Keywords-Leaf Water Content; Vegetation Index; Hyperspectral

\section{INTRODUCTION}

Leaf water content is an important parameter of describing vegetation growth, and it is also one of the important indicators to evaluate whether the crops are lack of water.

Leaf water content of the traditional detection method mainly is drying. Because of the limitation of sampling, this method can't show the situation of crop water content of large area well. How to quickly obtain the large area crop water information has attracted more attention. Crop water content by means of remote sensing inversion has become a good solution.

The current crop leaf water content by remote sensing information extraction method probably can be divided into the following categories:

(1) Direct inversion by the moisture absorption band. Many studies have shown that in the region of the near infrared and short-wave infrared wavelengths, there are five moisture absorption bands in 970, 1200, 1450, 1930 and $1200 \mathrm{~nm}$. In the past, many models were constructed based on the relationships sensitive wave bands and the leaf water content ${ }^{[1-2]}$.

(2) Methods of spectrum analysis. Along with the continuously growing, crop growth parameters make a lot of changes, these changes cause the difference of crops spectrum. Because of these differences, making use of spectrum analysis technology can obtain much information of vegetation spectral characteristics. The characteristic parameters mainly are the characteristic of spectral absorption or reflection peak valley, such as absorption or reflection wavelength band location, depth, slope, width, symmetry, area and the reflectance ratio ${ }^{[3-}$ 4]

(3) Vegetation index. Vegetation indices (VIs) are the combination of multiband reflectance. In the means of monitoring vegetation conditions by remote sensing, vegetation indices are more reliable than a single band. Due to good correlations between vegetation leaf water content and shortwave infrared and near infrared band, many researchers have used the two band area of the reflectance to construct vegetation indexes to extract of leaf water content information. Common used moisture of vegetation index such as NDWI, MSI, WI, PWI, GVMI ${ }^{[5]}$.

(4) Methods of Vegetation water content ratio index. Canopy spectra mainly influenced by three major factors: leaf spectra, vegetation canopy structure and the observation Angle sensor. Since most sensor use the vertical projection, so a lot of research focused in two aspects of leaf and canopy structure. For Leaf water content of inversion, the main consideration is the cross influence between the leaf area index (LAI) and leaf water content. How to come up an index which is sensitive to leaf water content, but not sensitive to LAI, has become a difficult problem. Ratio index method has shown a good way to solve this ${ }^{[6]}$.

(5) Radiative transfer models. The study found that the canopy reflectance model combined with leaf optical model can be used for inversion of leaf water content. The coupling model can be made for estimating leaf and canopy parameters, then estimate leaf EWT by canopy reflectance spectra ${ }^{[2]}$.Now several common radiative transfer models are used, such as PROSPECT, LIBERTY, SAIL, KUUSK ${ }^{[7-8]}$.

Due to the unreliability of the experience model and difficult problem by using radiative transfer model inversion, vegetation index are now more and more applied in the inversion of vegetation water content. After the analysis of the crop moisture sensitive wave bands, the triangular vegetation moisture index was improved with using compound ratio index. It is a new way to acquire leaf water content information.

*Corresponding author 


\section{MODIFIED TRIANGLE WATER VEGETATION INDEX}

Du (2013) proposed a new Vegetation Index of Vegetation Water content - Triangle Water Vegetation Index (Triangle Water Vegetation Index, TWI), which can be used to retrieve crop canopy Water content ${ }^{[9]}$.But in the actual agricultural research, individual leaf water content is more required. To achieve this requirement, we have done some improvement. Now Let's see how the TWI constructed.

\section{A. Triangle Water Vegetation Index(TWI)}

As we all know, there are three obvious vegetation moisture absorption areas in the spectral range $350-2500 \mathrm{~nm}$. But two areas of $1420 \mathrm{~nm}$ and $1960 \mathrm{~nm}$ centered are vulnerable to the effects of atmospheric water vapor content, so it's hard to makes use of the two bands in the remote sensing application because of too much noise. Many researches focused in the area of $970 \mathrm{~nm}$.

The field observations of canopy spectra at $970 \mathrm{~nm}$ is characterized integrated performance of leaf area index and crop leaves absorb moisture characteristics. In order to further understand the crop water at $970 \mathrm{~nm}$ absorption characteristics, the study simulated canopy spectrum under different leaf water content by the way of Prospect + SAIL. As shown in figure 1 was the canopy spectra with the use of PROSPECT + SAIL software simulation in the leaf area index of 2, leaf water content were $0.01,0.02,0.03,0.04,0.05,0.06,0.07$, $0.08,0.07,0.08\left(\mathrm{mu} \mathrm{g} / \mathrm{cm}^{2}\right)$, and other parameters were set at the default.

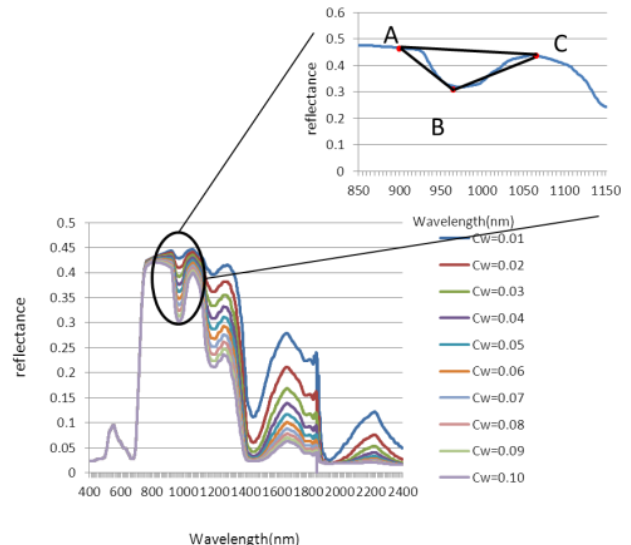

Figure 1 Canopy spectra under different leaf water content

From the simulated spectrum, we can see that due to the absorption of moisture, vegetation spectrum has an obvious absorption valley between $900 \mathrm{~nm}$ to $1070 \mathrm{~nm}$. As the increasing of vegetation water content, the absorption valley became more and more deeper. According to the observed phenomena, Du designed triangle moisture index to measure the size of the absorption valley. Specific principle is shown in figure 1. Point A, B and C represents the band reflectance in the $900 \mathrm{~nm}, 900 \mathrm{~nm}$ and $1070 \mathrm{~nm}$. When moisture percentage increasing, the reflectance of Point B $(970 \mathrm{~nm})$ decreasing, the reflectance of $\mathrm{A}$ and $\mathrm{C}$ are low correlation with vegetation moisture and reflectance basic remain unchanged, the area of the triangle $\mathrm{ABC}$ is increasing conversely. As vegetation reduced moisture percentage, an opposite phenomenon can be seen. So it's a good way to make use of the phenomenon. Through calculation, the final design of triangular vegetation. The water content index expression is shown as (1).

$$
T W I=S_{\triangle} A B C=0.5 \times\left(70 \times\left(R_{1070}-R_{900}\right)-170 \times\left(R_{970}-R_{900}\right)\right)(1)
$$

where $\mathrm{S} \triangle \mathrm{ABC}$ represents the area of the triangle $\mathrm{ABC}$, R1070, R970 and R900 represents the reflectance of $1070 \mathrm{~nm}$, $970 \mathrm{~nm}$ and $900 \mathrm{~nm}$ respectively.

\section{B. The improvement of Triangle Water Vegetation Index}

Monitoring crop canopy water content with TWI has good effect, but the performance for the extraction of leaf water content information is unsatisfactory [9]. This is mainly because of the influence of leaf area index. Ratio index is a good way to solve this problem. The modified soil adjustable vegetation index was introduced to construct a new index.

MSAVI is an improved one based on the soil vegetation index (SAVI). MSAVI can monitor the changes of leaf area index, also can reduce the effect by soil background. The formula of MSAVI is shown below.

$$
\text { MSAVI }=0.5\left[2 R_{750}+1-\sqrt{\left(2 R_{750}+1\right)^{2}-8\left(R_{750}-R_{680}\right)}\right]
$$

Where $R_{750}, R_{680}$ represents the reflectance of $750 \mathrm{~nm}, 680$ nm respectively.

Eventually Modified Triangle Water Vegetation Index (M TWI) is shown in formula (3).

$$
\text { M-TWI= TWI/MSAVI }
$$

\section{THE RESULTS AND DISCUSSION}

In order to verify the relation between M-TWI with vegetation water, the PROSPECT + SAIL model simulation data and field observation data were analyzed.

\section{A. Validation and discussion with simulated data}

PROSPECT model is a leaf optical model based on the radiative transfer theory developed in Allen plate-type model theory. It is used to calculate the visible and near-infrared wavelengths (400 $\mathrm{nm}$ to $2500 \mathrm{~nm}$ ) hemisphere reflectance and transmittance. SAIL model is the most mature and efficient canopy spectral model, which focus in the problem of the canopy multiple scattering. Due to mainly research equivalent water thickness EWT, so we just set equivalent water thickness and LAI. The specific input parameters are shown in table 1.

Table 1 Prospect + SAIL model input parameters

\begin{tabular}{cc}
\hline Input parameters & Value \\
\hline EWT $\left(\mathrm{mg} / \mathrm{cm}^{2}\right)$ & $0.01,0.02,0.03,0.04,0.05,0.06,0.07,0.08$, \\
$\mathrm{LAI}$ & $0.09,0.10$ \\
$\mathrm{~N}$ & $0.01,0.1,0.5,1.0,1.5,2.0,3.0,4.0,5.0,6.0$ \\
$\mathrm{Cm}\left(\mathrm{g} / \mathrm{cm}^{2}\right)$ & 1.3 \\
$\mathrm{Ch}\left(\mathrm{mg} / \mathrm{cm}^{2}\right)$ & 0.005 \\
$\mathrm{LAD}$ & 40 \\
\hline
\end{tabular}


Figure 2 shows the correlation between the M-TWI which calculated from Prospect + SAIL simulation data and EWT. It can be seen that the figure in a scatter diagram represents regularity. The linear correlation of new index-MTWI is better than the result of the TWI which made by DU. The fitting precision is up to 0.5927 . But the study found that when LAI < 0.5 , the relationship between $\mathrm{M}$ - TWI and leaf water content was very poor, if the points LAI $<0.5$ were removed, the linear relation model fitting precision can reach 0.80 as shown in figure 2 (b), the degree of discrete has further improved, so the new complex ratio vegetation index - TWI can be well used to monitor leaf water content, especially under the condition of high vegetation coverage.

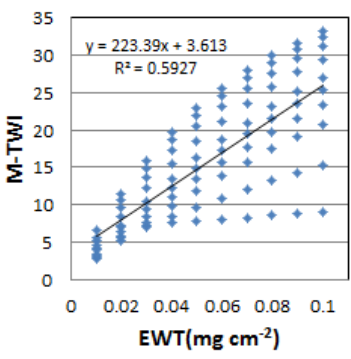

a

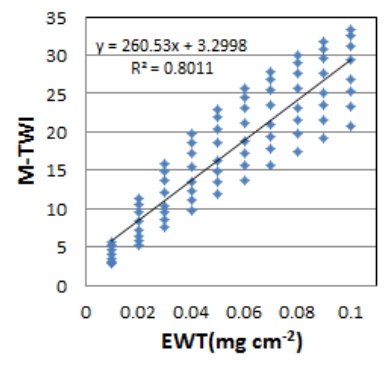

b
Figure 2 Correlation analysis between M-TWI and leaf water content

\section{B. Validation and discussion with measured data}

In order to further verified M - TWI in the actual application situation, the observed data of wheat which came from water and nitrogen coupling proving ground in Yucheng Shandong were used. The testing ground is composed of 32 experimental plots. The plot is a $10 \mathrm{~m} \mathrm{x} 5 \mathrm{~m} * 1 \mathrm{~m}$ (length $\mathrm{x}$ width $\mathrm{x}$ height) cement pool. Treating with the different nitrogen and irrigation, the plots were divided into 10 kinds. Every kind has three samples. In the whole growth period, the wheat of spectral data and leaf water content were got by sampling observation. The relations between the index and the measured leaf water content of EWT are shown in figure 3. From the figures, it can be found that the result is similar to the result of simulation data by PROSPECT + SAIL. TWI represented very poor, especially in high water content conditions. And M - TWI has good correlation, data clearly present linear distribution, similar to the simulation results.

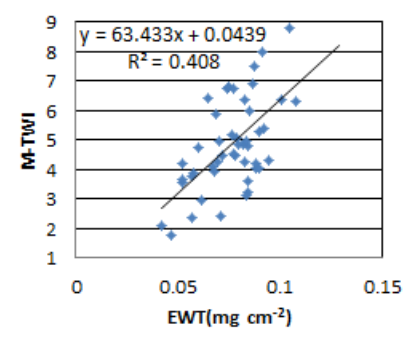

a

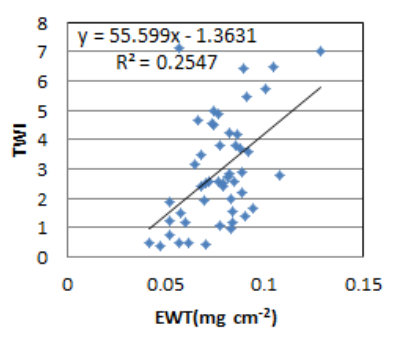

b
Figure 3 Relationships between M-TWI (a), TWI (b) and the leaf water content

From above research results, a conclusion can be made the new index (M - TWI) has a good correlation with leaf water content, so M - TWI can be used to monitor leaf water content.

\section{CONCLUSION}

According to the characteristics of leaf water content, a new index was modeled based on simulation and field observation data. Although the results of the study show good, but there are still some deficiencies need to be further improved in future studies.

First of all, a lot of researches in this article are based on PROSPECT + SAIL two physical models, but these models can't fully simulate realistic reaction conditions. Because leaf is described as a multi- Leaf Biochemistry uniform flat in PROSPECT, and Canopy is composed of the same leaf collections which has the same biochemical component. But there are many kinds of crops, while different crops have different leaf biochemical component distributions. So it needs more data to validate model, and establish a more stable model.

Secondly, in the establishment of the relationships between moisture and the vegetation index, we used mathematical regression method, which is lack of physical meaning.

Finally, our study only selected wheat samples with homogeneous ecological condition, the model can get a good retrieval accuracy. In reality, there are a lot of mixed pixels with the spaceborne remote sensing data, such as mixing different types of vegetation pixels, mixing soil and vegetation pixels and so on. These may affect the accuracy of the model to extract the information of vegetation water content. Due to the lack of relevant data, so there is no use of spaceborne hyperspectral, which needs to be done in the future.

\section{ACKNOWLEDGMENT}

This research is financed by the Natural Science Foundation of Hubei (GrantNo.2014CFB858), the Foundation Research Funds for the Central Universities (WUT: 2014-IV075, 2014-ZH-B1-05) and the Research Project of Hubei Provincial Department of Education (B2014278).

\section{REFERENCES}

[1] G. Eason, B. Noble, and I.N. Sneddon, "On certain integrals of Lipschitz-Hankel type involving products of Bessel functions," Phil. Trans. Roy. Soc. London, vol. A247, pp. 529-551, April 1955. (references)

[2] Curran P.J, Dungan J.L., Maeler B.A.Plummer S.E.,\&Peterson D.L Reflectance spectroscopy of fresh whole leaves for the estimation of chemical composition. Remote Sensing of Environmen, vol.39, pp. 153166, 1992.

[3] Ceccato P, Gobron N, Flasse S, et al. Designing a spectral index to estimate vegetation water content from remote sensing data: Part 1 Theoretical approach. Remote Sens Environ, vol. 82, pp. 188-197, 2002.

[4] Kokaly R,Clark R N. Determination od leaf chemical concentration using band-depth analysis of absorption features and stepwise linear regression. Remote sensing of Environment, vol. 67, pp. 267-287, 1999.

[5] ZhangJ H, Xu Y, Yao F M, et al. Advances in estimation methods of vegetation water content based on optical remote sensing techniques. Sci China Tech Sci,vol. 40, pp. 1121-1129, 2010.

[6] Tian Q, Tong Q, Pu P, et al. Spectroscopic determination of wheat water status using 1650-1850nm spcetral absorption feature. Int J Remote Sens, vol. 22, pp. 2329-2338, 2001,

[7] Jacquemoud S, Baret F. PROSPECT: a model of leaf optical properties spectra. Remote Sens Environ, vol. 34, pp. 75-91, 1990. 
[8] Verhoef W. Light scattering by leaf layers with application to canopy reflectance modeling: The SAIL model. Remote Sens Environ, vol. 16, pp. 125-141, 1984.

[9] Iaquinta J, Pinty B. Adaptation of a bidirectional reflectance model including the hot-spot to an optically thin canopy. In: Proceedings of the 6th International Symposium on Physical Measurements Signatures in Remote Sensing, Val d'Ise `re, France, pp. 683-690, 1994.

[10] Du Chen, Meng Qingye, Qin Qiming, Dong Heng.The development of a new model on vegetation water content.2013 IEEE International Geoscience and Remote Sensing Symposium(IGARSS), pp.3277-3280, 2013. 